\title{
Healing of Tendon Defects Implanted with a Porous Collagen-GAG Matrix: Histological Evaluation
}

\author{
L.K. LOUIE, Ph.D., ${ }^{1,5}$ I.V. YANNAS, Ph.D., ${ }^{2}$ H.-P. HSU, M.D., ${ }^{3,4}$ and \\ M. SPECTOR, Ph.D. ${ }^{2,3,4}$
}

\begin{abstract}
There is currently no method to restore normal function in tendon injuries that result in a gap. The objective of this study was to evaluate the early healing of tendon defects implanted with a porous collagen-glycosaminoglycan (CG) matrix, previously shown to facilitate the regeneration of dermis and peripheral nerve. A novel animal model that isolates the tendon defect site from surrounding tissue during healing was employed. This model used a silicone tube to entubulate the surgically produced tendon gap of $10 \mathrm{~mm}$, allowing for the evaluation of the effects of the analog of extracellular matrix on healing of tendon, absent the influences of the external environment. The results showed that tendon stumps induced synthesis of a tissue cable inside the silicone tube in both the presence and absence of CG matrix. The presence of the CG matrix, however, altered the process of tendon healing. Tubes filled with CG matrix contained a significantly greater volume of tissue at the time periods of evaluation: 3, 6, and 12 weeks. Granulation tissue persisted for a longer period of time in the lesion site of CG-filled defects, and the amount of dense fibrous tissue increased continuously during the period of study in defects filled with CG matrix. In contrast, the amount of dense fibrous tissue decreased after 6 weeks in originally empty tubes. In tubes that did not contain the CG matrix, the new tissue consisted of dense aggregates of crimped fibers with a wavelength and fiber bundle thickness that were significantly shorter than those in normal tendon, and consistent with the type of scar that is the end result of repair of many connective tissues. Although, CG-filled tubes contained dense fibrous tissue by 12 weeks, the tissue had no crimp. The CG matrix may have prolonged the synthesis of granulation tissue and delayed or prevented the formation of scar.
\end{abstract}

Departments of ${ }^{1}$ Materials Science and Engineering, and ${ }^{2}$ Mechanical Engineering, Massachusetts Institute of Technology, Cambridge, MA 02139.

${ }^{3}$ Rehabilitation Engineering R\&D Laboratory, Brockton/West Roxbury VA Medical Center, West Roxbury, MA 02132 .

${ }^{4}$ Department of Orthopedic Surgery, Brigham and Women's Hospital, Harvard Medical School, Boston, MA 02115.

${ }^{5}$ Current Address: Department of Materials Science and Engineering University of Michigan Ann Arbor, MI 48109.

Presented at the Inaugural Meeting of the Tissue Engineering Society, Orlando, Florida, December 13-15, 1996. 


\section{INTRODUCTION}

$\mathbf{R}$ UPTURE OF TENDONS can lead to life-long disability. Healing of these tissues depends on a) the extent of the injury, b) the type of surrounding connective tissue available to contribute to the healing process, c) the degree to which the ends of the tendon can be approximated, d) the capability of the cells in tendon to migrate into and proliferate and differentiate in the wound site, and e) the type of motion imparted to the site during the healing process. It remains uncertain how these factors interact and affect the healing process. Moreover, it has been difficult to determine the intrinsic capability of tendon to heal for most animal models and human observations, because of the extrinsic influence of surrounding tissues. Healing of tendon has generally been found to proceed by way of "repair," that is, the formation of scar tissue. Despite the fact that, under some circumstances, this scar can assume some of the cellular, biochemical, histological, and biomechanical features of normal tendon, healing does not result in full recovery of tendon composition and function. Tendon regeneration requires the migration, proliferation, and differentiation of cells in the wound site, such that the phenotype and distribution of cells within the healed substance would be as found in normal tendon. These considerations have prompted the development of an analog of extracellular matrix, comprising a porous collagen-glycosaminoglycan (CG) polymer, to serve as a scaffold to facilitate the regeneration of tendon at sites at which the substance has been lost. The importance of this approach to engineer tendon derives, in part, from the problems encountered in using other methods to treat tendon injuries: direct suture, prosthetic replacements, and tissue grafts.

Considerable attention has been directed toward methods of suturing tendon lacerations and the beneficial effects of continuous passive motion on healing of this tissue. Several techniques of suturing have been proposed to increase the immediate strength of the repair, and to facilitate subsequent healing. ${ }^{1-4}$ The challenge in obtaining a suture repair of a tendon injury increases significantly the greater the segmental loss of tissue. Suturing techniques alone are inadequate for the treatment of these injuries in which a gap exists between the ends of the tendon.

Generally, there has been an indication that postoperative mobilization significantly enhances the healing of tendon injuries. Studies have shown that tendons mobilized during healing differ considerably morphologically from tendons that were immobilized during healing. ${ }^{5-7} \mathrm{~A}$ thickened epitenon and cellular Iayer migrating into the depths of the repair site were found in the groups undergoing mobilization. Other studies have shown an increase in the load-carrying capacity in tendons that have undergone mobilization during healing. It is important to note, however, that many of these animal models employed Iacerations of the tendon, without much of a segmental loss of tissue.

The search for a prosthesis to replace tendons has paralleled approaches used for ligament replacement, and has been prompted by the desire to obtain immediate load-bearing capability. ${ }^{8-12}$ Devices fabricated from synthetic polymers and carbon fibers have been investigated in animal and human subjects. Problems generally related to the insertion of the device into bone, proper tensioning, and issues related to the abrasion of the prosthesis against bone at sites where the prosthesis exited a tunnel through bone. While encouraging results were reported in the short term, questions about the long-term performance of these devices limited their use. Considering that polymers have been found to undergo biodegradation and mechanical breakdown in other applications, expectations for their long-term performance as tendon prostheses should be limited.

The role of soft tissue autografts and allografts in treating tendon injuries is still uncertain. Questions relate to the source of the donor graft, methods of attachment to the residual tendon fragments, and proper tensioning. These grafts are generally used in situations where there is a desire to have immediate postoperative mobilization. However, many of these soft tissue grafts are unable to provide adequate long-term function, and do not adequately serve as templates for regeneration of the injured tendon.

In an effort to develop a fibrous template for tendon regeneration, Kato et al. ${ }^{13}$ investigated a carbodiimide-cross-linked collagen-fiber prosthesis. In studies in which this implant was used to bridge a gap in the Achilles tendon of rabbits, the prosthesis yielded results similar to those of autogenous tendon grafts, in that neotendon replaced the implants; the neotendon, however, was "similar, but not identical, to normal tendon," 1 year after implantation of the prosthesis.

The rationale in this study for use of an CG analog of extracellular matrix to facilitate regeneration of 


\section{HEALING OF TENDON DEFECTS IMPLANTED WITH A POROUS COLLAGEN-GAG MATRIX}

tendon was based, in part, on the success experienced in developing such substances for the regeneration of dermis ${ }^{14-17}$ in animals and human subjects and the reconnection of axons of cells in ruptured peripheral nerves in rats. ${ }^{16,18,19}$ Moreover, there was an indication that such an approach was successful in the regeneration of meniscus. ${ }^{20}$ Left untreated, defects in these tissues would not heal by regeneration. The present investigation employed a novel rabbit animal model that isolated a $10-\mathrm{mm}$ tendon gap from surrounding tissue by entubulation of the tendon stumps in a silicone tube; the model allowed for the evaluation of the effects of a CG matrix on healing of the tendon, absent the influences of the external environment.

\section{MATERIALS AND METHODS}

A gap was produced surgically in the right Achilles tendons of 24 rabbits. Under general anesthesia and aseptic condition, the posterior aspect Achilles tendon was exposed using a straight longitudinal incision. The Achilles and plantaris tendons were retracted posteriorly to expose the anterior area of the Achilles tendon. The tendons of the peroneus long, bevis and tertius were cut and a portion of each tendon was dissected away. This procedure was performed to immobilize the tendon gap site. The plantaris tendon was cut with a "Z" plasty, and the Achilles tendon transected at its middle. The Achilles tendons stumps immediately retracted about 8 to $10 \mathrm{~mm}$. The wound area was kept covered and moist with a wet saline soaked gauze while the knee joint was immobilized by external fixation to reduce loading to the tendon. Maintaining the hock joint at 45-degree extension, the tendon stumps were inserted into a silicone tube, with or without the CG contents (see below). A 10-mm gap in the tendon was maintained by three modified Kesslertype stitches through each stump and the tube, that allowed any mechanical stresses imparted to the system to be taken up by the sutures. The two " $Z$ " surfaces of the tendon of plantaris were separated away, then the touched parts were sutured under minimum tension. The three time periods of evaluation were 3 , 6 , and 12 weeks, with four animals receiving an empty tube and four rabbits implanted with a tube filled with the CG matrix at each time period.

The CG matrices consisted of type I bovine hide collagen precipitated from acid dispersion with chondroitin-6-sulfate as previously described. ${ }^{21}$ The suspended coprecipitate suspension was injected into a silicone tube ( $3.8 \mathrm{~mm}$ inside diameter) and immersed into a coolant bath and freeze-dried, to produce the porous CG matrices. The matrices were then exposed to a dehydrothermal treatment for cross-linking and sterilization. The matrix was nominally $95 \%$ porous with an average pore channel diameter of 60 $\mu \mathrm{m} .{ }^{22}$

At sacrifice, the excised tendons were fixed in $10 \%$ neutral buffered formaldehyde. The silicone tube was split lengthwise to expose the contents. Specimens were sectioned into three parts: proximal tissue (which included tissue of the proximal tendon stump) midsection tissue (which was composed of reparative tissue), and distal tissue (which included tissue of the distal stump). The contralateral tendon was similarly sectioned. The tissues were dehydrated and embedded in paraffin, and microtomed sections stained with hematoxylin and eosin for cell composition and Masson's trichrome for organization and orientation of the collagenous extracellular matrix. Tissue sections stained with hematoxylin and eosin were viewed with polarized light microscopy to evaluate the crimp pattern in the collagenous extracellular matrix. The wavelength, or period, of the crimp was measured, as was the fiber bundle thickness, the thickness of the bundle of collagen fibers that indulated together to form the crimp pattern.

\section{RESULTS}

Grossly, within the silicone tube, there appeared to be a cable of tissue spanning the wound gap for all specimens, except for a few specimens that had "pull-out" of the tendon stump, and were omitted from the study. While the amount of tissue within the silicone tubes decreased with time, there was consistently more reparative material in the tubes that had initially contained the CG matrices than those implanted empty. Histologically, by 12 weeks, tissue in the empty tube and tube filled with CG matrix had decreased from $100 \%$ of the defect to $5 \%$ and $15 \%$ of the wound site, respectively. 
$\mathbf{A}$

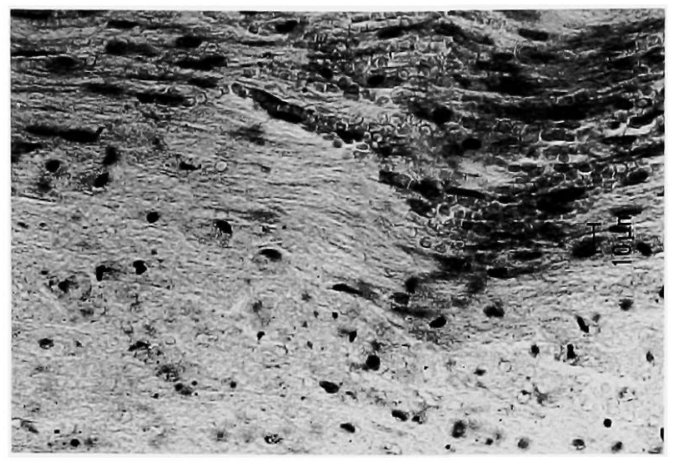

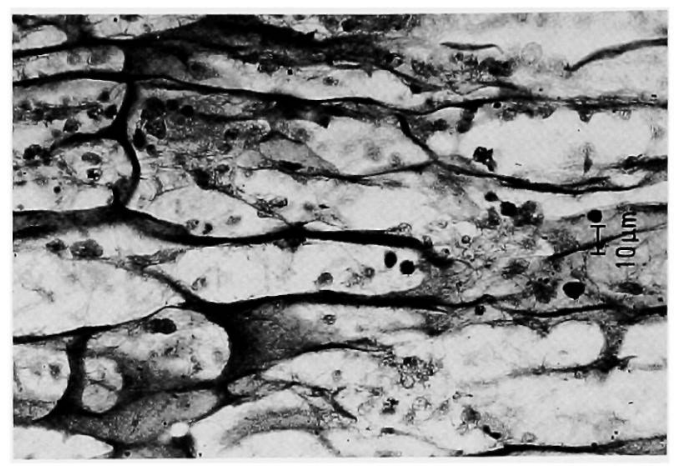

FIG. 1. Light micrographs of tissue in the middle of defects treated with an empty silicone tube (A) and a tube filled with the collagen-GAG (CG) matrix (B), taken 3 weeks postoperatively. Neovascular granulation tissue was found in both treatment sites, including within the CG matrix (B). H\&E stained paraffin sections.

\section{Three Weeks}

Inflammatory and fibroblast-like cells, and new blood vessels, were readily identifiable in the animals that received the empty silicone tubes (Fig. 1A). The fibroblasts were rounded with large nuclei. The cellular and extracellular make-up of the reparative tissue at this time period was consistent with granulation tissue. Masson's trichrome revealed loose fibrous tissue with regions of densely packed collagen bundles. The loose fibrous tissue appeared to be randomly orientated (ie, no preferential fiber direction) but the denser-packed fibers appeared to be aligning in the long direction of the tendon. The dense fibrous tissue

A
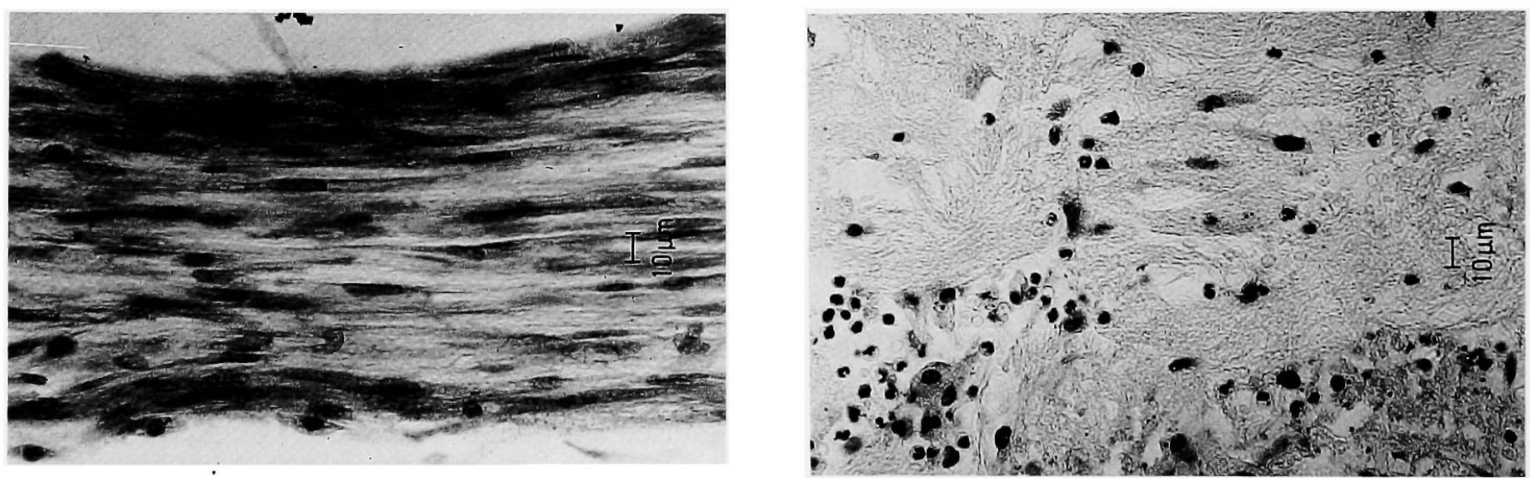

C

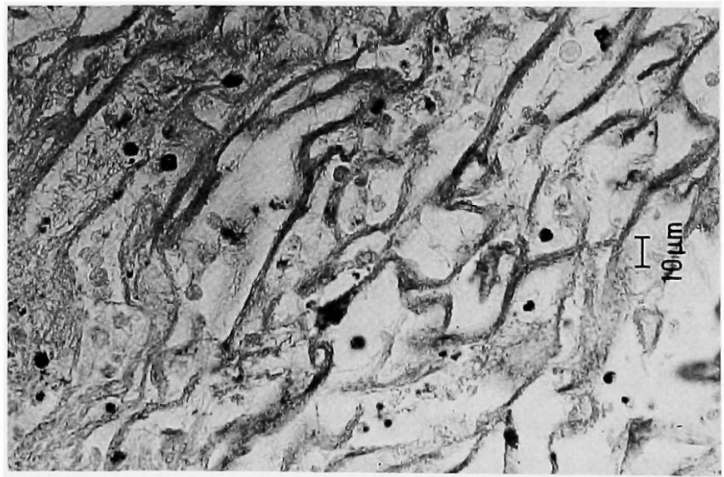

FIG. 2. Histology of 6-week tissue in the empty silicone tube (A) and the tubes filled with the CG matrix $(B, C)$. The empty tube contained predominantly loose fibrous tissue, much of which was aligned along the long axis of the tendon (A). Granulation tissue persisted in the tubes filled with the CG matrix (B), and some residual matrix could be found in the defect (C). (A-C) H\&E stained paraffin sections. 


\section{HEALING OF TENDON DEFECTS IMPLANTED WITH A POROUS COLLAGEN-GAG MATRIX}

was localized at the outer regions of the lumen of the tube, whereas the central portion of the defect contained loosely organized and randomly orientated fibers.

CG matrices were found at this postoperative period with no indication of resorption (Fig. 1B). Inflammatory cells and blood products predominated in tissue in CG-matrix-filled tubes, and infiltrated the CG matrix. Fibroblast-like cells were not as clearly identifiable in the 3-week samples. Masson trichrome revealed a loose fibrous matrix with localized regions of aligned dense fibrous matrix fibers in the CG filled tubes. There was in general more cellular material present in the CG-filled tube than the empty tubes. As in the empty tubes, dense aligned matrix fibers were located toward the outer zone of the lumen.

None of the fibrous tissue found in the empty and CG-filled tubes displayed a crimp pattern.

\section{Six Weeks}

The reparative material in the tubes implanted without the CG matrix was predominantly fibrous (Figs. $2 \mathrm{~A}$ and $3 \mathrm{~A}, \mathrm{~B})$, in contrast to the granulation tissue found earlier. The majority of the cells in the empty tubes were fibroblastic, displaying a more elongated morphology than cells in the 3-week tissue. The spindle-shaped fibroblasts were randomly oriented in the defect. The nuclei of the cells appeared to be smaller

A

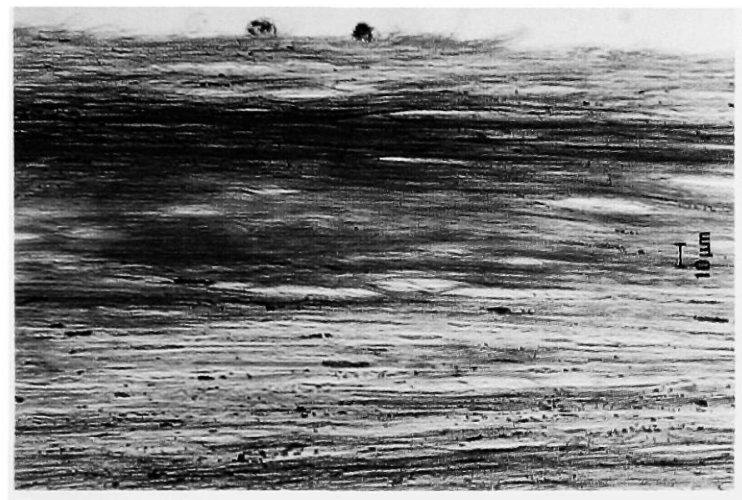

C

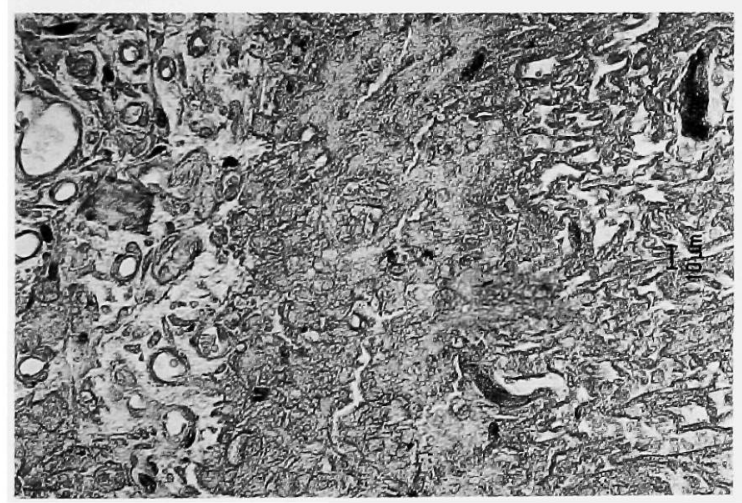

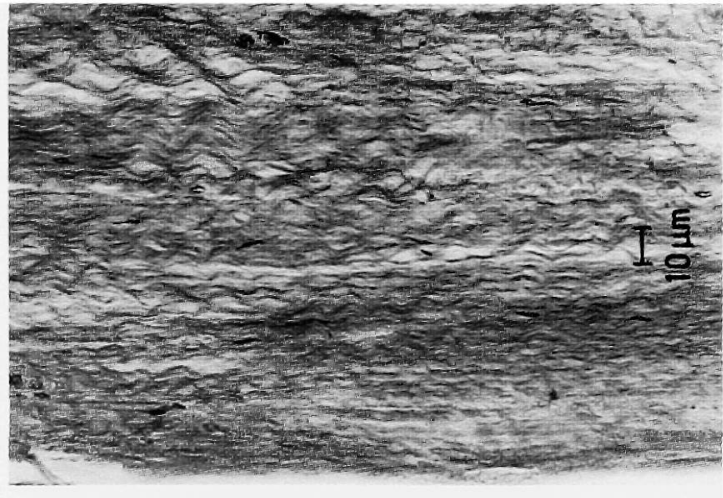

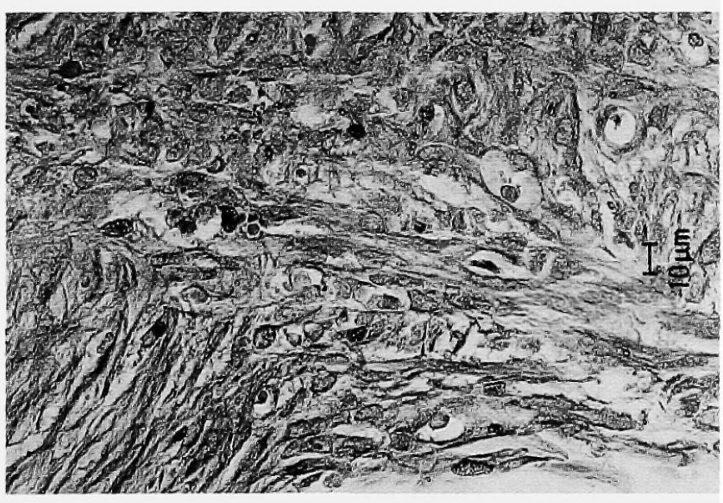

FIG. 3. Histology of 6-week tissue in the empty silicone tube $(A, B)$ and the tubes filled with the CG matrix $(C, D)$. The empty tube contained predominantly loose fibrous tissue, much of which was aligned along the long axis of the tendon (A,B). Granulation tissue persisted in the tubes filled with the CG matrix and some residual matrix could be found in the defect $(C)$. The fibers of the reparative tissue in the empty tube generally stained positive for collagen with Masson trichrome (A,B), but there were areas that did not stain as collagen-bottom of (A). Some of the densely packed collagenous tissue, aligned along the long axis of the tendon, displayed no crimp (A), while other tissue demonstrated crimp (B). Some of the extracellular matrix of the loosely arranged reparative tissue in the tube with the CG matrix stained lightly for collagen-left of (C). Except for a few areas dispersed throughout the sponge, the CG matrix itself did not stain with the Masson trichrome stain-right of (C). A woven network of collagen (D) could be seen in tubes with the CG matrix, adjacent to the tendon stump; the area in (D) is from the same tube as (C). (A-D) Masson trichromestained paraffin sections. 


\section{LOUIE ET AL.}

than at 3 weeks. The central portion of the defect contained fibers, that stained positive with a collagen stain (Masson trichrome; Fig. 3A,B), aligned along the long axis of the tendon; however, there remained many regions in which the fibers were not as highly aligned. In some regions, the densely packed collagen was crimped (Fig. 3B).

Tissue in the tubes with the CG matrix had the appearance of granulation tissue (Fig. 2B, and Fig. 3C). While still very cellular, it was less so than at 3 weeks. CG material was present in some areas of the wound site (Figs. 2C, 3C), although some resorption of the matrices was evident. The majority of the inflammatory cells appeared to be in the same regions as the CG matrices. Fibroblasts were present toward the edge of the tissue. They appeared rounded to slightly elongated with relatively large nuclei. In general, the reparative tissue fibers were aligned in the long direction of the tendon. Also, the central portion of the tissue, that did not appear to have aligned fibers at 3 weeks, now displayed isolated regions with oriented fibrous tissue. A more woven collagenous extracellular matrix was found in the tubes adjacent to the tendon stumps (Fig. 3D).

None of the fibrous tissue in the CG-filled tubes displayed a crimp pattern. One of the empty tube specimens had small regions that displayed a crimp pattern. These regions were confined to the outer border of the tissue sample.

\section{Twelve Weeks}

The majority of fibroblasts in the empty silicone tubes had become highly elongated with a further decrease in the size of the nuclei (Fig. 4A). The cells and particularly the extracellular collagen fibers (Fig.

A

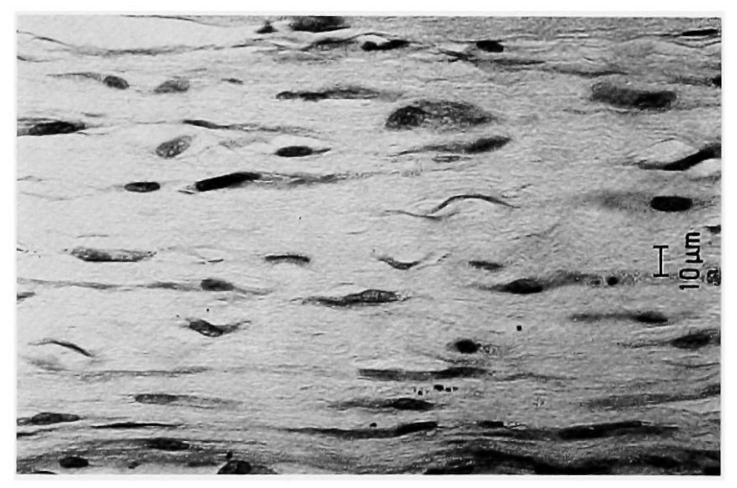

C

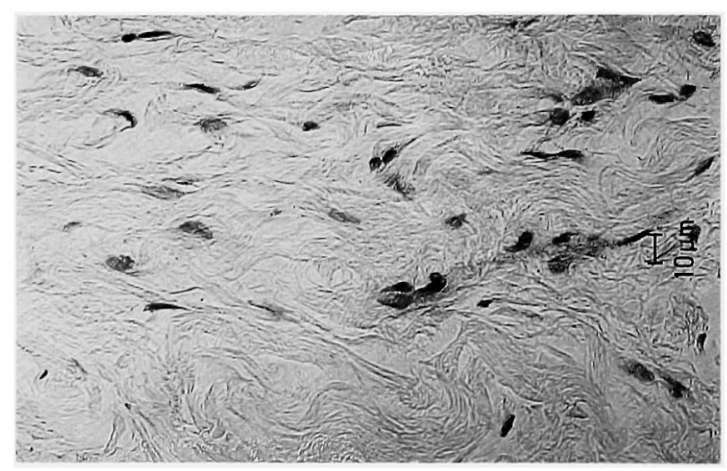

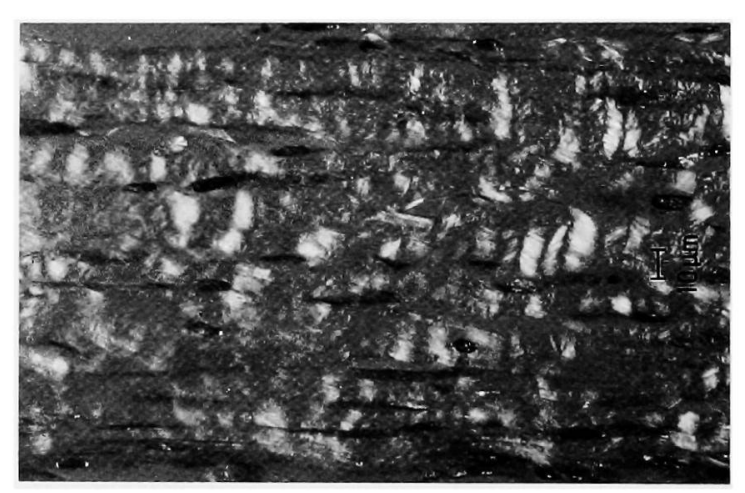

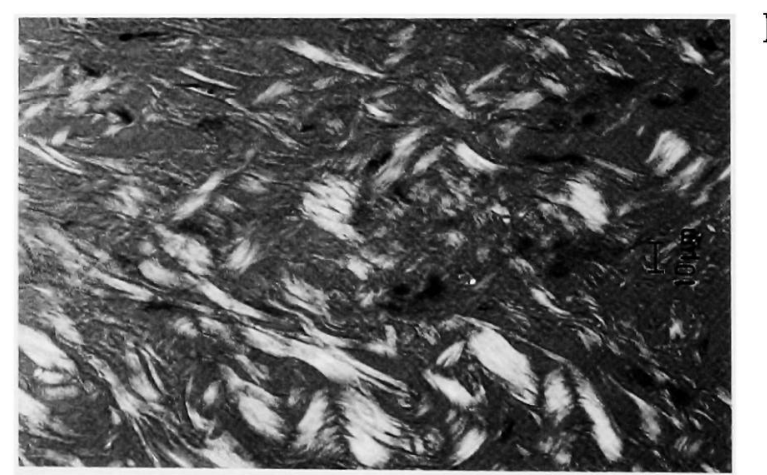

FIG. 4. Micrographs of tissue in the middle of defects treated with the silicone tube alone (A,B) and a tube filled with the CG matrix (C,D), taken 12 weeks postoperatively. The polarized light micrographs in (B) and (D) are of the same areas as shown in (A) and (C). The collagen fibers and spindle-shaped fibroblasts in tube implanted without the CG matrix are aligned along the long axis of the tendon (A), and the collagen fibers display a crimp pattern (B) with a wavelength approximately one-tenth that of normal tendon (see Fig. 4 for comparison). The collagen fibers in the defect implanted with the CG matrix (D) do not display the crimp pattern seen in the defect reacted with the empty tube (B). Paraffin sections sections stained with H\&E. 
$4 A, B)$ were highly aligned in the long direction of the tendon. The overall number of cells also appeared to have decreased from 6 weeks.

The number of cells in the CG-filled tube decreased significantly (Fig. 4C), and there was no evidence of CG matrix present; resorption of the matrices appeared to have occurred between 6 and 12 weeks. Fibroblasts were the predominant cell. They ranged from slightly elongated to highly elongated with smaller cell nuclei. In general, cells toward the edges appeared to be more highly elongated and aligned in the long direction of the tendon, with the cells toward the center of the tissue slightly less elongated, and not necessarily aligned in the long direction of the tendon. The tissue in tubes filled with CG matrix comprised of collagen fibers aligned along the tendon axis and others randomly oriented (Fig. 4C,D).

Tissue in empty tubes had more regions with crimp pattern than at earlier time periods (Fig. 4B). The crimp wavelength was $12.2 \pm 3.0 \mu \mathrm{m}$ (average \pm SEM) and the fiber bundle thickness was $30.2 \pm 11.0$ $\mu \mathrm{m}$ (Fig. 4B). Normal tendon had a crimp wavelength of $110 \pm 20 \mu \mathrm{m}$ and fiber bundle thickness of $1.2 \pm$ $0.4 \mathrm{~mm}$ (Fig. 5). Fibrocollagenous tissue in tubes filled with collagen-GAG matrices displayed some areas in which the collagen fibers were folded, but did not demonstrate a crimp pattern (Fig. 4D).

\section{DISCUSSION}

Tendon healing results in the formation of scar, which differs morphologically, biochemically, and biomechanically from the original tissue. While this scar tissue may assume some of the characteristics of tendon, complete regeneration does not occur. Questions remain about the origin of the cells responsible for the production of collagen in this reparative tissue and the mechanism by which the originally disorganized collagen fibers align themselves and adopt the normal crimp pattern. Controversy surrounding the source of the cells relates to the intrinsic capability to heal ${ }^{23}$ versus the extrinsic contribution from surrounding tissues or from the tendon sheath or the epitenon ${ }^{24-26}$; and some believe that both intrinsic and extrinsic sources of collagen-producing cells contribute to the healing process. ${ }^{25,27}$ A drawback of the studies supporting intrinsic healing is that they were performed in vitro; conclusive support of intrinsic healing in animal experiments is difficult because of the problem of differentiating between collagen-producing cells originating from the tendon and those cells originating from extratendinous tissue.

The present study is the first investigation of the healing of an entubulated tendon gap that isolated the defect both biologically and mechanically from the external environment during healing. The results showed that tendon stumps induced synthesis of tissue capable of bridging a 10-mm gap in the presence and absence of CG matrix. The presence of the CG matrix, however, altered the kinetics of tendon healing. Tubes filled with CG matrix contained a significantly greater volume of tissue at all time periods. At 3 and 6 weeks, the presence of the nondegraded CG matrix may have contributed, in part, to the volume, but no residual CG matrix was observed at 12 weeks. Granulation tissue persisted for a longer period in the lesion

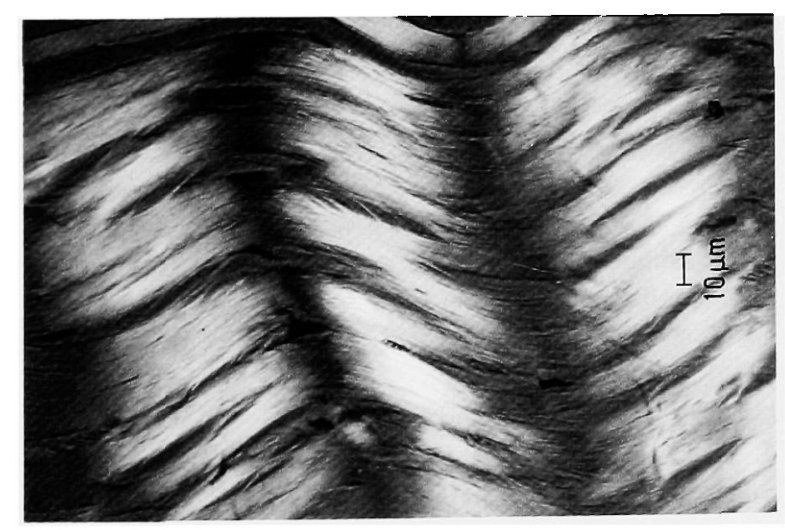

FIG. 5. Polarized light micrograph of normal tendon displaying the characteristic crimp pattern. Paraffin sectioned stained with H\&E. 


\section{LOUIE ET AL.}

site of CG-filled matrices. The amount of dense fibrous tissue increased continuously during the period of study in defects filled with CG matrix; in contrast, the amount of dense fibrous tissue decreased after 6 weeks in the originally empty lesions. Moreover, in empty tubes, by 6 weeks, some of the fibrous tissue comprised dense aggregates of crimped fibers with a wavelength and fiber bundle thickness that were both significantly shorter than those in normal tendon. Although, CG-filled tubes contained dense fibrous tissue, by 12 weeks, the tissue had no crimp. The CG matrix may have prolonged the synthesis of granulation tissue and have affected the ability of the matrix fibers in the tissue to align.

The crimp pattern of tendon has been shown to play an important role in its mechanical properties. ${ }^{28,29}$ The wavelength and thickness of fiber bundles associated with the crimp of the dense fibrous tissue found in the empty tubes were significantly smaller than for normal tendon. Similar results have been reported by Kato and associates ${ }^{13,30,31}$ in studies of the response of tendon healing to the presence of resorbable collagen-based fibrous grafts. The tissue in Kato's tendon lesion site was described to have a crimp wavelength $(10 \mu \mathrm{m})$ comparable with that of tissue in the empty tube at 12 weeks described in this study $(12 \mu \mathrm{m})$. In Kato's study, it was also observed that this crimp pattern was present from 3 weeks to 52 weeks with minimal change in the crimp characteristics. The question that arises: Is this an end-stage scar tissue? That the tissue in Kato's study did not appear to remodel significantly by 52 weeks, and is similar to tissue found in the empty tube at 12 weeks in this study, leads one to believe that this tissue may be terminal "scar" tissue. Moreover, the crimp pattern was consistent with scar tissues found in other healing tissues. These findings indicate that the CG matrix altered the process of tendon healing such that the formation of scar was delayed or prevented. If this is the case, the results would parallel the findings of studies in which a CG matrix delayed the onset of, and reduced the severity of, scarring in full-thickness dermal wounds, and thereby facilitated regeneration. ${ }^{17}$

The present study has shown that an animal model can be implemented to investigate the course of healing of a relatively wide gap in a large tendon, absent extrinsic influences. Spontaneous healing through 12 weeks results in a small diameter cable of fibrous tissue with crimp characteristics consistent with scarwith a crimp wavelength approximately one order of magnitude smaller than that of normal tendon. The presence of a CG matrix modifies the reparative process, apparently delaying or preventing scar formation. This work serves as a foundation for future longer term studies to determine the extent to which tendon regeneration is achieved with the CG matrix alone, or with cell-seeded contructs that may accelerate the process of regeneration.

\section{ACKNOWLEDGMENT}

This work was funded in part by the Brigham Orthopedic Foundation.

\section{REFERENCES}

1. Becker, H. Primary repair of flexor tendons in the hand without immobilisation: Preliminary report. Hand, 10, 37, 1978.

2. Seradge, H. Elongation of the repair configuration following flexor tendon repair. J. Hand Surg. 8, $182,1983$.

3. Wade, P.J.F., Muir, I.F.K., and Hutcheon, L.L. Primary flexor tendon repair: The mechanical limitations of the modified Kessler technique. J. Hand Surg. 11B, 71, 1986.

4. Wray, R.C., and Weeks, P.M. Experimental comparison of technics of tendon repair. J. Hand Surg. 5, $144,1980$.

5. Gelberman, R.H., Manske, P.R., Akeson, W.H., Woo, S.L-Y., Lundborg, G., and Amiel, D. Flexor tendon repair, J. Orthop. Res., 4, 119, 1986.

6. Gelberman, R.H., Woo, S.L-Y., Lothringer, K., Akeson, W.H., and Amiel, D. Effects of early intermittent passive mobilization on healing canine flexor tendons. J. Hand Surg. 7A, 170, 1982.

7. Gelberman, R.H., Nunley, J.A., Osterman, A.L., Breen, T.F., Dimick, M.P., and Woo, S.L-Y. Influences of the protected passive mobilization internal on flexor tendon healing: A prospective randomized clinical study. Clin. Orthop., 264, 189, 1991. 


\section{HEALING OF TENDON DEFECTS IMPLANTED WITH A POROUS COLLAGEN-GAG MATRIX}

8. Amis, A.A., Campbell, J.R., and Miller, J.H. Strength of carbon and polyester fibre tendon replacements. Variation after operation in rabbits. J. Bone Joint Surg. 67, 829, 1985.

9. Aragona, J., Parsons, J.R., Alexander, H., and Weill, A.B. Soft tissue attachment of a filamentous carbon absorbable polymer tendon and ligament replacement. Clin. Orthop. 160, 268, 1981.

10. Foster, I.W., Ralis, Z.A., McKibbin, B., and Jenkins, D.H.R. Biological reaction to carbon fiber implants: The formation and structure of carbon induced "neotendon." Clin. Orthop. 131, 299, 1978.

11. Mendes, D.G., Iusim, M., Angel, D., Rotem, A., Mordehovich, K., Roffman, M., Lieberson, S., and Boss, J. Ligament and tendon substitution with composite carbon fiber strands. J. Biomed. Mater. Res. 20, 699, 1986.

12. Rueger, J.M., Siebert, H.R., Wagner, K., and Pannike, A. Long-term implantation of a polyethelene ligament/tendon allograft in sheep. In: Christel, P., Meunier, A. and Lee, A.J.C., eds. Mechanical and histological studies, Biological and Biomechical Performance of Biomaterials. Amsterdam: Elsevier Science Publishers B.V., 1986, p. 135.

13. Kato, Y.P., Dunn, M.G., Zawadksy, J.P., Tria, A.J., and Silver, F.H. Regeneration of Achilles tendon with a collagen tendon prosthesis: Results of a one-year implantation study. J. Bone Joint. Surg. 73-A, 561, 1991.

14. Burke, J.F., Yannas, I.V., Quinby, W.C., Bondoc, C.C., and Jung, W.K. Successful use of a physiologically acceptable artificial skin in the treatment of extensive burn injury. Ann. Surg. 194, 413, 1981.

15. Yannas, I.V. Certain biological implications of mammalian skin regeneration by a model extracellular matrix. Cutaneous Develop. Aging and Repair 18, 131, 1989.

16. Yannas, I.V. Regeneration of skin and nerves by use of collagen templates. In: Nimni, M., ed. Collagen, Vol. III: Biotechnology. Boca Raton, FL: CRC Press, 1989, p. 87.

17. Yannas, I.V., Burke, J.F., Orgill, D.P., and Skrabut, E.M. Wound tissue can utilize a polymeric template to synthesize a functional extension of skin. Science 215, 174, 1982.

18. Chang, A.S., Yannas, I.V., Perutz, S., Loree, H., Sethi, R.R., Krarup, C., Norregaard, T.V., Zervas, N.T., and Silver, J. Electrophysiological study of recovery of peripheral nerves regenerated by a collagen-glycosaminoglycan copolymer matrix. Gebelein, C.G., ed. Progress in Biomedical Polymers. New York: Plenum Press, 1990, p. 107.

19. Yannas, I.V., Orgill, D.P., Silver, J., Norregaard, T.V., Zervas, N.T., and Schoene, W.C. Regeneration of sciatic nerve across $15 \mathrm{~mm}$ gap by use of a polymetric template. In: Gebelein, C.G., ed. Advances in Biomedical Polymers. New York: Plenum Press, 1987, p. 1.

20. Stone, K.R., Rodkey, W.R., Webber, R.J., McKinney, L., and Steadman, J.R. Future directions: Collagen-based prostheses for meniscal regeneration. Clin. Orthop. 252, 129, 1990.

21. Yannas, I.V., Lee, E., Orgill, D.P., Skrabut, E.M., and Murphy, G.F. Synthesis and characterization of a model extracellular matrix which induces partial regeneration of adult mammalian skin. Proc. Natl. Acad. Sci. U S A, 86, 933, 1989.

22 Louie, L.K., Yannas, I.V., and Spector, M. Development of a collagen-GAG copolymer implant for the study of tendon regeneration. In: Mikos, A.G., Murphy, R.M., Bernstein, H., and Peppas, N.A., eds. Biomaterials for Drug and Cell Delivery. Pittsburgh, PA: MRS, 1994, p. 19.

23. Manske, P.R. Intrinsic flexor-tendon repair. J. Bone Joint. Surg. 66A, 385, 1984.

24. Buck, R.C. Regeneration of tendon. J. Pathol. Bact. 66, 1, 1953.

25. Garner, W.L., and McDonald, J.A. Identification of the collagen-producing cells in healing flexor tendon. Plast. Reconstr. Surg. 83, 875, 1989.

26. Lindsay, W.K. The fibroblast in flexor tendon healing. Plast. Reconstr. Surg. 34, 223, 1964.

27. Russell, J.E., and Manske, P.R. Collagen synthesis during primate flexor tendon repair in vitro. J. Orthop. Res. 8, 11,1990

28. Dale, W.C., and Baer, E. Fibre-buckling in composite systems: A model for the ultrastructure of uncalcified collagen tissues. J. Mater. Sci. 9, 369, 1974.

29. Comninou, M., and Yannas, I.V. Dependence of stress-strain nonlinearity of connective tissues on the geometry of collagen fibers. J. Biomechanics 9, 427, 1976.

30. Wasserman, A.J., Kato, Y.P., Christiansen, D., Dunn, M.G., and Silver, F.H. Achilles tendon replacement by a collagen fiber prosthesis: Morphological evaluation of neotendon formation. Scanning Microsc. 3, 1183, 1989.

31. Goldstein, J.D., Tria, A.J., Zawadsky, J.P., Kato, Y.P., Christiansen, D., and Silver, F.H. Development of a reconstituted collagen tendon prosthesis. J. Bone Joint Surg. 71-A, 1183, 1989.

Address reprint requests to:

Libby Louie, Ph.D.

Department of Materials Science and Engineering

University of Michigan

Dow Bldg.

Ann Arbor, MI 48109-2136 


\section{This article has been cited by:}

1. Victor S. Nirmalanandhan , Jason T. Shearn , Natalia Juncosa-Melvin, Marepalli Rao , Cynthia Gooch , Abhishek Jain , Gino Bradica, David L. Butler . 2008. Improving Linear Stiffness of the Cell-Seeded Collagen Sponge Constructs by Varying the Components of the Mechanical StimulusImproving Linear Stiffness of the Cell-Seeded Collagen Sponge Constructs by Varying the Components of the Mechanical Stimulus. Tissue Engineering Part A 14:11, 1883-1891. [Abstract] [PDF] [PDF Plus]

2. Natalia Juncosa-Melvin, Karl S. Matlin, Robert W. Holdcraft, Victor S. Nirmalanandhan, David L. Butler . 2007. Mechanical Stimulation Increases Collagen Type I and Collagen Type III Gene Expression of Stem Cell-Collagen Sponge Constructs for Patellar Tendon RepairMechanical Stimulation Increases Collagen Type I and Collagen Type III Gene Expression of Stem Cell-Collagen Sponge Constructs for Patellar Tendon Repair. Tissue Engineering 13:6, 1219-1226. [Abstract] [PDF] [PDF Plus]

3. Natalia Juncosa-Melvin, Jason T. Shearn, Gregory P. Boivin , Cynthia Gooch , Marc T. Galloway , John R. West , Victor S. Nirmalanandhan, Gino Bradica, David L. Butler . 2006. Effects of Mechanical Stimulation on the Biomechanics and Histology of Stem Cell-Collagen Sponge Constructs for Rabbit Patellar Tendon RepairEffects of Mechanical Stimulation on the Biomechanics and Histology of Stem Cell-Collagen Sponge Constructs for Rabbit Patellar Tendon Repair. Tissue Engineering 12:8, 2291-2300. [Abstract] [PDF] [PDF Plus]

4. Natalia Juncosa-Melvin, Gregory P. Boivin , Marc T. Galloway, Cindi Gooch , John R. West , David L. Butler . 2006. Effects of Cell-to-Collagen Ratio in Stem Cell-Seeded Constructs for Achilles Tendon RepairEffects of Cell-to-Collagen Ratio in Stem Cell-Seeded Constructs for Achilles Tendon Repair. Tissue Engineering 12:4, 681-689. [Abstract] [PDF] [PDF Plus]

5. Natalia Juncosa-Melvin, Gregory P. Boivin, Cynthia Gooch, Marc T. Galloway, John R. West, Michael G. Dunn , David L. Butler . 2006. The Effect of Autologous Mesenchymal Stem Cells on the Biomechanics and Histology of Gel-Collagen Sponge Constructs Used for Rabbit Patellar Tendon RepairThe Effect of Autologous Mesenchymal Stem Cells on the Biomechanics and Histology of Gel-Collagen Sponge Constructs Used for Rabbit Patellar Tendon Repair. Tissue Engineering 12:2, 369-379. [Abstract] [PDF] [PDF Plus]

6. Patty Chen , Erika Marsilio , Ronald H. Goldstein , Ioannis V. Yannas , Myron Spector . 2005. Formation of Lung Alveolar-Like Structures in Collagen-Glycosaminoglycan Scaffolds in VitroFormation of Lung Alveolar-Like Structures in Collagen-Glycosaminoglycan Scaffolds in Vitro. Tissue Engineering 11:9-10, 1436-1448. [Abstract] [PDF] [PDF Plus]

7. Natalia Juncosa-Melvin, Gregory P. Boivin, Marc T. Galloway, Cynthia Gooch, John R. West, Angela M. Sklenka , David L. Butler . 2005. Effects of Cell-to-Collagen Ratio in Mesenchymal Stem Cell-Seeded Implants on Tendon Repair Biomechanics and HistologyEffects of Cell-to-Collagen Ratio in Mesenchymal Stem Cell-Seeded Implants on Tendon Repair Biomechanics and Histology. Tissue Engineering 11:3-4, 448-457. [Abstract] [PDF] [PDF Plus]

8. Randell G. Young, David L. Butler, Wade Weber, Arnold I. Caplan, Stephen L. Gordon, David J. Fink. 1998. Use of mesenchymal stem cells in a collagen matrix for achilles tendon repair. Journal of Orthopaedic Research 16:4, 406-413. [CrossRef] 